Konjunkturpaket

\section{Mehr Platz für Kitas und Schulen!}

Um nach den Sommerferien Schüler*innen bundesweit Präsenzunterricht zu ermöglichen, muss laut Bundesbildungsministerin Karliczek (CDU) der Unterricht notfalls in Containern stattfinden. Klar ist, dass dies nur möglich ist, wenn es der Pandemieverlauf erlaubt. Klar ist auch, dass dies ein Vorstoß ist, um endlich konkrete Möglichkeiten des Präsenzunterrichts auf den Weg zu bringen. Abstandsregelungen sind einzuhalten, deshalb bedarf es mehr Räumen, wenn in kleineren Gruppen unterrichtet wird. Aber Lernen findet nicht erst in der Schule statt, sondern schon in der Kita. Deshalb brauchen auch Kitas Container bzw. zusätzliche Räume! Auch hier muss in kleineren Gruppen gearbeitet werden. Mehr Räume sind wichtig und zwar vom Anfang der Bildungskette an.

Karliczek und Bundesfamilienministerin Giffey (SPD) sollten sich am Kabinettstisch dafür stark machen. Sie müssen mit Bundesfinanzminister Scholz (SPD) verbindliche Absprachen treffen, wenn es um Bundesmittel geht. Der Bund darf im Bereich der Investitionskosten für Kitas und Schulen investieren, zumindest in beschränktem Umfang. So sind im Konjunkturpaket auch $3 \mathrm{Mrd}$. Euro für diesen Bereich vorgesehen. Dies sind $2 \%$ der insgesamt $130 \mathrm{Mrd}$. Euro. Doch mehr Räume alleine reichen nicht, denn es gibt auch mehr Bedarf an Lehrenden und an pädagogischem Fachpersonal, zumindest dann, wenn Curricula nicht massiv gekürzt werden sollen, nicht ganze Bereiche der frühen Bildung wegfallen sollen. Hier sollte man sich am Gesundheitsbereich orientieren und diejenigen heranziehen, die gerade ausgebildet werden. In diesem Fall: angehende pädagogische Fachkräfte für Kitas oder Lehramtsanwärter für Schulen. Sie könnten einen Teil ihrer erforderlichen Praxiserfahrung jetzt sammeln, um nicht nur eine räumliche, sondern auch personelle Entzerrung zu ermöglichen. Bekannt ist, dass der Bund hier nicht direkt investieren darf, sondern die Länder und Kommunen gefordert sind. Länder und Kommunen müssen entlastet werden, damit sie investieren können.

„Maßnahmen, die die Konjunktur möglichst schnell ankurbeln, oder Zukunftsinvestitionen?", war eine der Schlüsselfragen für das Konjunkturpaket. Investitionen in Lernräume und Lehrpersonal sind beides. Denn die Bildung und Betreuung in Kitas und der Präsenzunterricht

(C) Der/die Autor(en) 2020. Open Access: Dieser Artikel wird unter der Creative Commons Namensnennung 4.0 International Lizenz (https:// creativecommons.org/licenses/by/4.0/deed.de) veröffentlicht.

Open Access wird durch die ZBW - Leibniz-Informationszentrum Wirtschaft gefördert. in Schulen ermöglicht es Alleinerziehenden und erwerbstätigen Eltern, Familien- und Berufsarbeit zu vereinbaren. Das sind immerhin zwei Drittel der Familien mit Kindern bis zwölf Jahre. Insbesondere Mütter mit Kindern in dieser Altersgruppe können gegenwärtig wegen TeilschlieBungen von Kitas und Schulen nicht voll erwerbstätig sein oder ihre Produktivität leidet, zumindest dann, wenn sie sich noch weiter so „durchwurschteln" müssen. Das heißt, den Unternehmen fehlen nicht nur Arbeitskräfte, sondern dem Fiskus Einnahmen aus Einkommensteuer und Sozialversicherungen. Aber auch die Produktivität von Eltern und insbesondere Müttern mit älteren Kindern kann leiden, wenn ältere Kinder keinen Präsenzunterricht bekommen. Denn nicht alle Kinder über zwölf Jahren lernen (nur) selbstständig, also ganz ohne Präsenzunterricht. Auch das beeinflusst das Wohlbefinden von Familien. Einbußen im Wohlbefinden verursachen Kosten für die Volkswirtschaft - das „Bruttonationalglück“ sinkt und kann auch das Bruttoinlandsprodukt schmälern.

Der langfristige Charakter dieser Investitionen liegt in der Entzerrung von Raum und Personal: Unterlassene Schulbildung kostet den Einzelnen, die Gesellschaft und die Volkswirtschaft. Geht etwa ein Drittel eines Schuljahres verloren, so geht dies im Schnitt mit $3 \%$ bis $4 \%$ geringerem Lebenseinkommen einher.

Bildung hat auch vielfache nicht-monetäre Erträge. Nicht umsonst hat das Bundesbildungsministerium in den letzten Jahren dazu ein Forschungsprogramm aufgelegt. Schulbildung kann die physische Gesundheit verbessern, was wiederum den Bundesgesundheitsminister Spahn (CDU) interessieren sollte. Bildung erhöht das Demokratieverständnis und reduziert z. B. die Sorgen, die sich Menschen über die Einwanderung nach Deutschland machen. Frühkindliche Bildung hat ebenfalls eine hohe Rendite. Studien belegen, dass langfristig jeder investierte Euro mehrfach als Ertrag zurückkommen kann, wenn es sich um eine gute Bildung und Betreuung handelt. Andere Studien zeigen, dass insbesondere Kinder aus sozioökonomisch benachteiligten Familien davon profitieren. Belegt ist auch, dass ein Kita-Besuch ebenfalls die Integration der Eltern verbessern kann. Allerdings fallen diese Erträge erst in 25 bis 30 Jahren an. Das sind aber Investitionen in die Generationen, die die heute gemachten Schulden vermutlich zurückzahlen müssen. Deshalb sollten Giffey und Karliczek gemeinsam mit den Ländern und Kommunen innovativere Vorschläge einbringen. 